\title{
Defined Substance Administration Period Dose Total
}

National Cancer Institute

\section{Source}

National Cancer Institute. Defined Substance Administration Period Dose Total. NCI

Thesaurus. Code C93800.

Total of all doses of treatment in a given period of time (as defined in dosePeriodCode). 